\title{
Circadian pain assessment in neonates from a nurse’s perspective
}

\author{
Padysakova $\mathrm{H}$ \\ Faculty of Nursing and Professional Health Studies, Slovak Medical University Bratislava, Slovakia. \\ hana.padysakova@szu.sk
}

\section{ABSTRACT}

OBJECTIVES: Circadian rhythms modulate the activity of pain pathways. But there appears to be a lack of understanding of how the perception of pain varies in neonates during the day. To assess circadian variations in pain perception we analyzed intensities of pain at $3 \mathrm{~h}$ intervals in neonates (within 24 hours). We also followed up the nursing interventions practiced for pain reduction and their effectivity within a day.

METHODS: We have analyzed the clinical observations of pain in 16 neonates during 62 days of hospitalization ( with Mean $=3.87$, Min $=1$, Max = 19). The pain was assessed by a modification of the Comfort scale supplemented with Wong-Baker scale. Procedure used for acute pain elimination was administration of $24 \%$ sucrose to the neonates. This procedure was centralized and performed in an incubator or neonatal bed.

RESULTS: We confirmed statistically significant difference in pain scores in our group of neonates at 10:00 am, 4:00 pm, 4:00 am as compared to $10: 00 \mathrm{pm}$ ( $\mathrm{p}$ values being $0.02603 ; 0.02885,0.01673$ ). The pain score was highest at 10:00 pm (Mean = 18.86, Max $=34$ ) and at 1:00 am (Mean = 18.89, Max = 33). There was no significant difference in the pain score at 10:00 pm and 1:00 am $(p=0.2951)$.

CONCLUSION: The results of our studies provided further evidence of circadian rhythm in the pain scores in neonates. A better understanding of circadian control of pain in neonates can help promote a more frequent use of time-contingent pain treatment strategies to help improve neonates care (Tab. 1, Fig. 2, Ref. 28). Text in PDF www.elis.sk.

KEY WORDS: circadian rhythm, neonate, nursing care, pain, the Comfort scale.

\section{Introduction}

Up until the late 1980s, it was widely considered among clinicians that neonates cannot perceive pain due to the belief that they were undeveloped (1). The understanding of pathophysiological mechanisms of pain in the neonate has achieved significant progress. At present we know that neonates react to noxious stimuli, experience stress and display stereotypical pain-induced responses. A review (2) on the neonatal pain management confirms that skin receptors and sensory nerves around the mouth appear as early as the 7 week of gestation. The fetal hormonal stress response can be observed at 18 weeks of gestation (3). From 20 weeks, the cortex and thalamus-cortex connections are present (4). By 23-25 weeks, free nerve endings and spinal cord projections are fully mature (5) but specific areas of pain appear active from 35 to 37 weeks of pregnancy (6).

Neonates are more sensitive to pain than older infants, children, and adults (7), and this hypersensitivity is further exacerbated in

Faculty of Nursing and Professional Health Studies, Slovak Medical University Bratislava, Slovakia

Address for correspondence: $\mathrm{H}$. Padysakova, PhD, PhD, Faculty of Nursing and Professional Health Studies, Slovak Medical University Bratislava, Limbova 14, SK-833 03 Bratislava, Slovakia.

Phone: +421.2.59370840 preterm neonates (8). Descending pathways that inhibit incoming pain impulses do not mature until the last trimester, which increases the preterm infant's sensitivity to pain (9).

The prevention of pain in neonates should be the goal of all health professionals. Repetitive, prolonged, and poorly treated pain in the neonatal period has the potential for deleterious consequences and it can cause permanent neuroanatomical and behavioral abnormalities (7, 10-12). Pain can induce anxiety, increased sensitivity to future pain, decreased effectiveness of analgesics and consequently increased analgesic requirements (13-15). In future, the child may try to avoid going through the medical procedure because of pain expectations.

During hospitalization in a neonatal intensive care unit (NICU), neonates experience up to 14 painful procedures per day. Moreover, neonates who underwent a surgery for severe malformations or inflammatory complications need effective management of postsurgical pain $(16,17)$. There still appears to be a lack of understanding of how neonates perceive pain and the resulting adverse sequelae of untreated pain in neonates (12). Moreover, circadian rhythms modulate activity and function of peripheral and central neurons of the pain pathways. Knowledge regarding the establishment of infant circadian rhythms postnatally is not well recognized. To assess circadian variations in pain perception we analyzed intensities of pain at $3 \mathrm{~h}$ intervals in neonates (within 24 hours). We also followed up the nursing interventions practiced for pain reduction and their effectivity within a day. 


\section{Patients and methods}

This study was conducted in the NICU. The study group consisted of neonates after surgery and neonates after therapeutic hypothermia for asphyxia. We analyzed clinical observations in 16 hospitalized neonates. Their pain was pharmacologically attenuated by the physician's order. The total length of hospitalization of the 16 neonates was 62 days, an average of 3.87 days, Min = 1.0, Max $=19.0$, Median $=3.0$, Modus $=1.0$.

The incidence of pain in neonates was evaluated 8 times a day at regular 3 hour intervals. The pain score in neonates was in the standard, the variance of score values $7-19$. The score 20-40 requires pharmacotherapy by the doctor.

We identified the critical times, which showed significant increase in the pain score of the neonate.

We used a modified Comfort scale, which is designed to assess comfort and postsurgical pain in non-intubated and artificially ventilated children. This method has also been validated for use in premature neonates. We supplemented the method with the WongBaker scale. The information value of the scale is not absolute, because some changes on the face may also occur in situations, that are not caused by pain $(13,18)$.

In the study, we also used procedures from the guidelines for nursing interventions to eliminate pain in neonatal patients (13, $18,19)$. We applied combination of painful procedures, and pain elimination techniques: minimal interruption of the neonate, intervention in the incubator or neonatal bed, administration of 24 $\%$ concentration of sucrose to the tongue.

Statistical analysis: Student's t-test was used for analyzing statistical differences.

Study limitations: We could not include a control sample due to ethics issues.

\section{Results}

The highest Max pain score values were at night as seen in Figure 1, which shows the statistical differences in the pain score indicators. Average pain score in neonates at the different observation times is seen in Table 1 . The Mean and Median scores did not reach the critical value of 20 in the studied neonates, which is the criterion for increasing the dose of pharmacotherapy (Tab. 1). The pain score was lowest at 7:00 am as seen by the scores (Min $=12.00$, Median $=15.50$, Mean = 15.65, Max = 20.00).

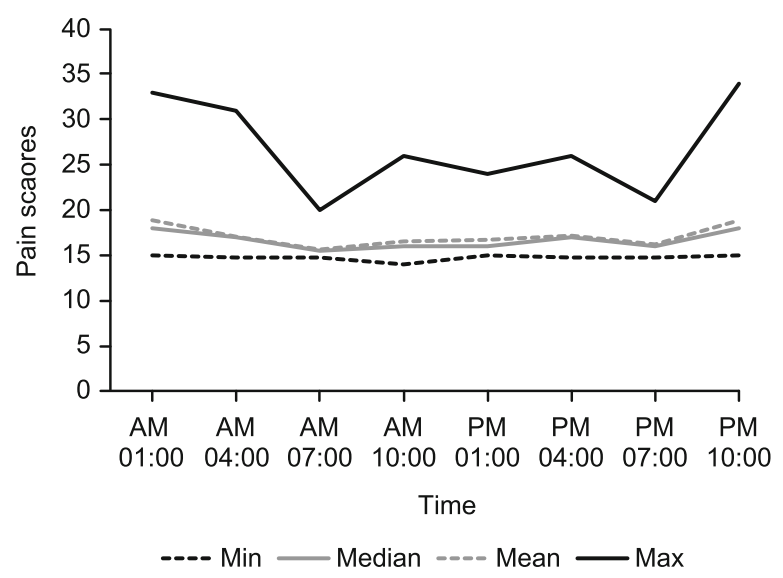

Fig. 1. Statistical pain score indicators.

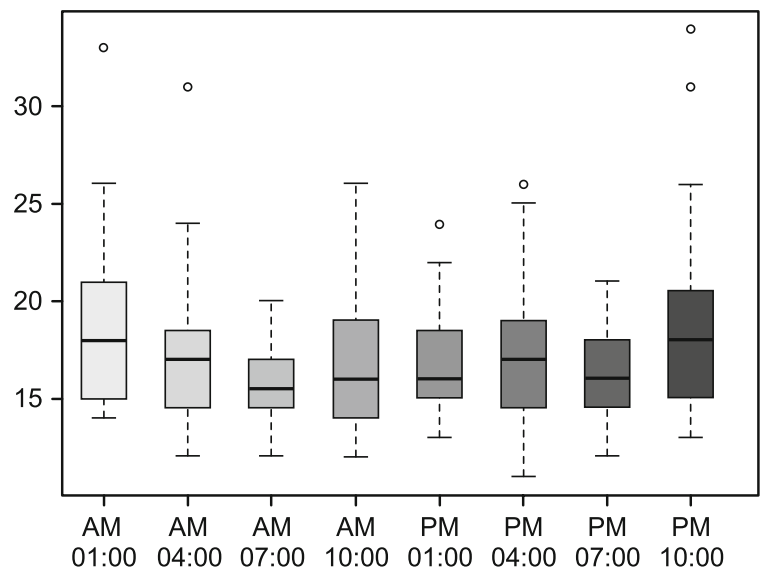

Fig. 2. Pain scores in neonates at different times of observation.

Statistically, we analyzed the pain score in the neonate sample at the time of observation (Fig. 2). From this table we can compare the pain score reached at 10:00 pm with highest recorded Max score and the scores at time intervals with a high Max value score - 4:00 am, 10:00 am, 4:00 pm, 1:00 am.

We used the Wilcoxon-Mann-Whitney rank sum test. At night time at 10:00 pm and at 1:00 am, we recorded the highest pain score in neonates. We found, that there is no statistically significant

Tab. 1. Average pain score in neonates at times of observation.

\begin{tabular}{|c|c|c|c|c|c|c|}
\hline \multirow{2}{*}{$\begin{array}{l}\text { Time for checking } \\
\text { the scores }\end{array}$} & \multicolumn{6}{|c|}{ Pain scores } \\
\hline & Min. & 1 st Qu. & Median & Mean & 3 rd Qu. & Max. \\
\hline AM 01:00 & 14.00 & 15.00 & 18.00 & 18.89 & 21.00 & 33.00 \\
\hline AM 04:00 & 12.00 & 14.75 & 17.00 & 17.07 & 18.25 & 31.00 \\
\hline AM 07:00 & 12.00 & 14.75 & 15.50 & 15.65 & 17.00 & 20.00 \\
\hline AM 10:00 & 12.00 & 14.00 & 16.00 & 16.54 & 19.00 & 26.00 \\
\hline PM 01:00 & 13.00 & 15.00 & 16.00 & 16.71 & 18.25 & 24.00 \\
\hline PM 04:00 & 11.00 & 14.75 & 17.00 & 17.18 & 19.00 & 26.00 \\
\hline PM 07:00 & 12.00 & 14.75 & 16.00 & 16.21 & 18.00 & 21.00 \\
\hline PM 10:00 & 13.00 & 15.00 & 18.00 & 18.86 & 20.25 & 34.00 \\
\hline
\end{tabular}


difference in pain score averages at 10:00 pm and at 1:00 am, $\mathrm{p}=$ 0.2951 . We compared pain scores in neonates at 10:00 am, 4:00 pm, 4:00 am and 10:00 pm. We found, that there is a statistically significant difference in pain score averages at 10:00 am and at $10: 00 \mathrm{pm}, \mathrm{p}=0.02603$, there is a statistically significant difference in pain score averages at $4 \mathrm{PM}$ and at 10:00 pm, p value $=0.02885$, there is a statistically significant difference in pain score averages at 4:00 am and 10:00 pm, p value $=0.01673$. We reject the hypothesis, that the pain score averages are the same at the observed intervals. Pain intensity in neonates is statistically significantly lower at 10:00 am, 4:00 pm and 4:00 am compared to 10:00 pm.

\section{Discussion}

In the present study we show significant differences in the intensity of neonatal pain at the observed intervals, valid for the study sample. We followed the basic guidelines for assessment of pain in children (19) which assesses the behavior of the child and their usual expressions; pays attention to the physiological signals that may indicate that the child feels pain; involves parents and/or personel who care and their observation of the normal behavior of the child and when the child is in pain the child normally behaves when in pain; assesses pain after pain-relieving interventions have been implemented and reassesses treatment; documents the child's pain assessment scores. The pain scores were evaluated using the Comfort scale supplemented with the Wong-Baker scale $(13,18)$. During painful procedures, $24 \%$ concentration of sucrose was administered to the tongue, which was effective. Meta-analysis studies $(2,20)$ have shown that doses of 0.24 g sucrose given 2 minutes before painful stimuli were effective for neonates and provided analgesia for 5-7 minutes.

Circadian rhythm is the approximately 24-hour cycle of change in the physiology of life. The infant is born with a weak sleepwake rhythm connected to the mother (21). The fetal biological clock generates circadian rhythms in response to maternal signals (activity, heart rate, body temperature, cortisol, melatonin), at least from the third quarter of pregnancy.

The neonate develops the components of circadian rhythm postnatally and the imprinting effects on the circadian rhythm persist into adulthood (21). But knowledge regarding the establishment of infant circadian rhythms is not well recognized.

Little is known about daily cycles of pain sensitivity. Endogenous opioids are important in modulating pain and it is suggested that circadian fluctuations in levels of these peptides can alter pain scores throughout the day. Circadian rhythms were also found to modulate mechanical thresholds, with reduced sensitivity immediately before the active phase relative to all other times in animal (22). However in our group of neonates we noticed that the pain score was lowest at 7:00 am (before active human phase) and pain intensity in neonates was statistically significantly lower at 10:00 am, 4:00 pm and 4:00 am compared to 10:00 pm (so-called the end of active phase). Our results are consistent with the findings that the highest sensitivity occurs during the evening in humans (23). But when we compare our results with studies in infants, they are not identical because several studies reported that in- fants in an awake state before acute pain displayed more robust behavioral and physiological reactivity when compared to those in quiet sleep states (9). But we observed the highest pain score in neonates at night time (10:00 pm and at 1:00 am). It should be taken under consideration that calculation of due date may be incorrect for planned births and there is an individual gestational age and intraindividual variability in maturing of organ systems (24).

Moreover, we cannot exclude that our results are distorted by unstable „undeveloped“ sleep-wake cycle in neonates. We consider the awakening of the child every 3 hours as a risk factor that increases pain.

Although pain experiences in the neonatal period are not consciously accessible to the individual, the plasticity of supraspinal foci involved in sensory processing supports the evidence that recurrent neonate pain alters the behavioral responses to pain in adulthood (25). Therefore, it is important for nurses to actively assess pain in neonates, to understand and score its intensity by using pain scales and to practice validated procedures for the prevention, reduction and assessment of pain. The first step in pain prevention is to plan nursing activities, so that the nurse minimizes the total number of painful procedures (2). Nursing interventions are to be carried out softly, gentle on the baby, they must necessarily take the shortest time and should be done in such a way that they do not multiply the discomfort of the child and do not induce secondary pain $(15,19,26)$. Various nonpharmacological treatments of pain that are recommended to provide neonates with are: non-nutritional suction, breastfeeding, administration of breast milk, sweet solutions, reduce illumination, skin-to-skin contact, kangaroo mother care, music therapy, and multisensorial stimulation.

The significant limitations of pain research, such as the inability of neonates to verbally vocalize if they are in pain can often lead to incorrect ideas. The nature of behavioral pain indicators can vary within each developmental stage of the neonate's life as well as between different gestational ages (9). An improvement in neonate's pain management, combined with the advances being made in sensor technology and artificial intelligence $(27,28)$, provide opportunities for research and innovation.

\section{Conclusion}

We have shown that there are significant differences in the intensity of neonatal pain at the observed intervals and we noticed the critical pain period in the nighttime. In NICU it is mandatory to provide individualized and humanized care. In this sense, nurses are qualified professionals for the development of pain evaluation and management protocols together with ongoing education actions. The results of our studies provided further evidence of circadian rhythm in pain scores in neonates. A deeper understanding of circadian control of pain in neonates can help promote more frequent use of time-contingent pain treatment strategies to help improve neonatal care. 
523-526

\section{References}

1. Owens ME, Todt EH. Pain in infancy: neonatal reaction to a heel lance. Pain 1984; 20 (1): 77-86.

2. Bhalla T, Shepherd E, Tobias JD. Neonatal pain management. Saudi J Anaesth 2014; 8 (1): 89-97.

3. Giannakoulopoulos X, Sepulveda W, Kourtis P et al. Fetal plasma cortisol and beta-endorphin response to intrauterine needling. Lancet 1994; 344 (8915): 77-81.

4. Garel C, Chantrel E, Brisse $\mathbf{H}$ et al. Fetal cerebral cortex: normal gestational landmarks identified using prenatal MR imaging. AJNR Am J Neuroradiol 2001; 22 (1): 184-189.

5. Fitzgerald M. Prenatal growth of fine-diameter primary afferents into the rat spinal cord: a transganglionic tracer study. J Comp Neurol 1987; 261 (1): 98-104.

6. Fabrizi L, Slater R, Worley A et al. A shift in sensory processing that enables the developing human brain to discriminate touch from pain. Curr Biol 2011; 21 (18): 1552-1558.

7. Anand KJ. Clinical importance of pain and stress in preterm neonates. Biol Neonate 1998; 73: 1-9.

8. Fitzgerald M, Millard C, MacIntosh N. Hyperalgesia in premature infants. Lancet 1988; 1 (8580): 292.

9. Ranger M, Johnston CC, Anand KJ. Semin Perinatol 2007; 31 (5): 283-288.

10. Grunau R. Early pain in preterm infants: a model of long-term effects. Clin Perinatol 2002; 29: 373-394.

11. Batton DG, Barrington KJ, Wallman C. Prevention and management of pain in the neonate: an update. Pediatrics 2006; 118: 2231-2241.

12. Anand KJ, Aranda JV, Berde CB et al. Summary proceedings from the neonatal pain-control group. Pediatrics 2006; 117 (3 Pt 2): S9-S22.

13. Stromer W, Messerer B, Crevenna R et al. Pain therapy for children and adolescents with hemophilia. Recommendations by an expert panel. Der Schmerz 2018; 32 (6): 404-418.

14. Newell A, Keane J, McGuire BE et al. Interactive Versus Passive Distraction and Parent Psychoeducation as Pain Management Techniques During Pediatric Venepuncture: A Randomized Controlled Trial. Clin J Pain 2018; 34 (11): 1008-1016.
15. Kristensen HN, Lundbye-Christensen S, Haslund-Thomsen H et al. Acute Procedural Pain in Children: Intervention With the Hospital Clown. Clin J Pain 2018; 34 (11): 1032-1038.

16. Kubricht V, Sencik $\mathbf{P}$. Chronic postsurgical pain in mixed surgical population. Bratisl Med J 2017; 118 (12): 746-751.

17. Mixa V, Nedomova B, Berka I. Continuous epidural analgesia, a new prospect in analgesia of newborns. Bratisl Med J 2015; 116 (9): 571-573.

18. Bansal N, Saha S, Jaiswal JN, Samadi F. Pain Elimination during Injection with Newer Electronic Devices: A Comparative Evaluation in Children. Int J Clin Pediatr Dent 2014; 7 (2): 71-76.

19. Twycross A, Dowden S, Stinson J (Eds). Managing Pain in Children: A Clinical Guide for Nurses and Healthcare Professionals. Oxford: WileyBlackwell, 2014: 112-178.

20. Taddio A, Shah V, Hancock $\mathbf{R}$ et al. Effectiveness of sucrose analgesia in newborns undergoing painful medical procedures. CMAJ 2008; 179 (1): 37-43.

21. Brooks E, Canal MM. Development of circadian rhythms: role of postnatal light environment. Neurosci Biobehav Rev 2013; 37 (4): 551-560.

22. Minett MS, Eijkelkamp N, Wood JN. Significant determinants of mouse pain behaviour. PLoS One 2014; 9 (8): e104458.

23. Hagenauer MH, Kile J, Piltz S et al. The modulation of pain by circadian and sleep-dependent processes: a review of the experimental evidence. In: Proceedings from A Research Collaboration Workshop for Women in Mathematical Biology. Berlin: Springer, 2016.

24. Hederlingova J, Psenkova P, Zahumensky J. The impact of physiological peripartal stress on the lifelong health of newborn. Bratisl Med J 2017; 118 (6): 324-327.

25. Anand KJ. Pain, plasticity, and premature birth: a prescription for permanent suffering? Nat Med 2000; 6 (9): 971-973.

26. McMurtry CM, Riddell RP, Taddio A et al. Far From „Just a Poke“: Common Painful Needle Procedures and the Development of Needle Fear. Clin J Pain 2015; 31 (10): 3-11.

27. El Hassoun O, Valaskova Z, Hulin I. Artificial intelligence opens new horizons in science and medicine and offers sophisticated solutions to complex problems. Cardiology Lett 2018; 27 (3): 153-161.

28. El-Hassoun O, Maruscakova L, Valaskova Z et al. Artificial intelligence in service of medicine. Bratisl Med J 2019; 120 (3): 218-222.

Received October 22, 2018. Accepted March 29, 2019. 